\title{
Effect of Wave Group Interactions on the Distribution of Wave Height
}

\author{
Yongquan $\mathrm{Li}^{*}$ \\ College of Oceanic and Atmospheric Sciences, Ocean University of China, 238 Songling Road, Qingdao 266100, P.R. China \\ *Corresponding author
}

\begin{abstract}
The study was made on the effect of wave group interaction on the distribution of wave height. The interaction of two wave groups was studied on the basis of coupled nonlinear Schrödinger equations. We found that wave group interaction may cause the deviation of wave height distribution from the result of linear theory. For low and medium wave heights, in the case of nonlinearity the probability of exceedence is higher than the result of linearity. For waves with large height, in the case of nonlinearity the probability of exceedence is lower than the result of linearity. From average wave height to 1.5 times this height is the transition region. The result is in consistency with that of wind wave experiment. A parameter was introduced in studying the influence of wave group interaction on wave height distribution. It is a measure of the effect of nonlinearity on the distribution. For wind waves this parameter reflects the shape of the spectrum. The spectral shape is found to be an important factor in influencing the distribution of wave height.
\end{abstract}

Keywords-wave height distribution; nonlinearity; numerical simulation; spectral shape

\section{INTRODUCTION}

The distribution of wave height is of interest both in theory and in ocean engineering. Longuet-Higgins [1] derived the distribution on the basis of linear theory and narrow spectrum assumption. In subsequent observations it was found that the wave height distribution deviated from the Rayleigh distribution [2-7]. It is now generally accepted that nonlinearity may cause the deviation from Rayleigh distribution and studies have been made in including wave steepness as a parameter in the distribution for engineering use [3]. However, hitherto there is no satisfactory theory which fully explains the deviation.

In the study of water waves Zakharov [8] proved that the evolution of the envelope of wave group is governed by nonlinear Schrödinger equation (NLS). NLS has since then been widely used in studies of modulation of water waves. In the case of two wave groups, the evolution of the envelope can be described by two coupled nonlinear Schrödinger equations (CNLS) [9-13]. CNLS has been used in stability problems in wave group evolution and it has been proved that under certain conditions the interaction of two wave groups can generate waves with very large wave height [10].

In this paper we study the effect of wave group interaction on the distribution of wave height. In section II we study the interaction between two wave groups on the basis of CNLS and show that the interaction may cause the deviation of wave height distribution from the result of linear theory. A parameter is introduced in studying the influence of wave group interaction on the distribution. In section III we study the distribution of wave height by wind wave experiment. The result of experiment is consistent with the result in section II. The introduced parameter in wave group interaction reflects the shape of the spectrum and is found to be an important factor in influencing the distribution of wave height. Section IV is conclusion.

\section{INTERACTION OF TWO WAVE GROUPS}

\section{A. Theoretical Analysis}

In this section we study the interaction between two wave groups and the effect of the interaction on the distribution of wave height.

Consider four simple sine waves with frequencies $\omega_{\mathrm{a}}, \omega_{\mathrm{b}}$, $\omega_{\mathrm{c}}, \omega_{\mathrm{d}}$. The difference between $\omega_{\mathrm{a}}$ and $\omega_{\mathrm{b}}$ is very small. The spectral value for these two waves is $s\left(\omega_{1}\right)$. In the same way, the difference between $\omega_{\mathrm{c}}$ and $\omega_{\mathrm{d}}$ is very small. The spectral value for these two waves is $s\left(\omega_{2}\right)$ (see Figure 1).

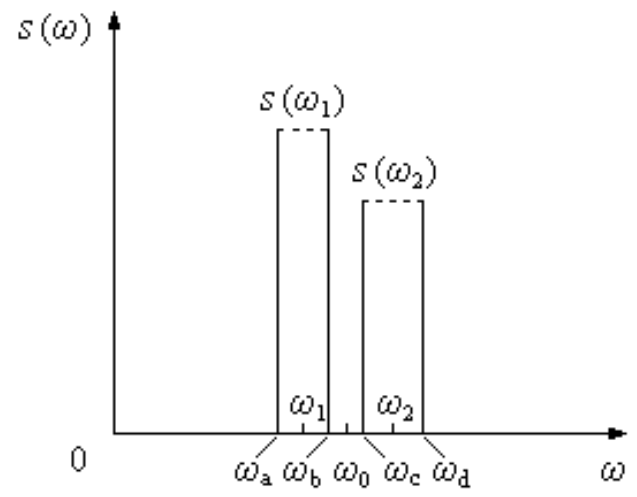

FIGURE I. SPECTRUM OF TWO WAVE GROUPS

The two waves with frequencies $\omega_{\mathrm{a}}$ and $\omega_{\mathrm{b}}$ compose one wave group. The two waves with frequencies $\omega_{\mathrm{c}}$ and $\omega_{\mathrm{d}}$ compose another wave group. When these two wave groups coexist, they interact with each other. Studies have been made on the interactions of wave groups in recent years. The evolution of the envelopes of the two wave groups is governed by CNLS $[9,10]$. For the two wave groups in Figure 1, when considering them propagating in one direction, CNLS can be written as 


$$
\begin{aligned}
& i\left(\frac{\partial A_{1}}{\partial t}+\frac{\partial \omega_{1}}{\partial k_{1}} \cdot \frac{\partial A_{1}}{\partial x}\right)+\alpha_{1} \frac{\partial^{2} A_{1}}{\partial x^{2}}+\varepsilon_{1}^{2}\left(\xi_{1}\left|A_{1}\right|^{2}+\zeta_{1}\left|A_{2}\right|^{2}\right) A_{1}=0 \\
& i\left(\frac{\partial A_{2}}{\partial t}+\frac{\partial \omega_{2}}{\partial k_{2}} \cdot \frac{\partial A_{2}}{\partial x}\right)+\alpha_{2} \frac{\partial^{2} A_{2}}{\partial x^{2}}+\varepsilon_{2}^{2}\left(\xi_{2}\left|A_{2}\right|^{2}+\zeta_{2}\left|A_{1}\right|^{2}\right) A_{2}=0
\end{aligned}
$$

where $A_{1}$ and $A_{2}$ are the envelopes of the two wave groups. $\varepsilon_{1}$ and $\varepsilon_{2}$ are the average steepness of the two envelopes.

$$
\begin{aligned}
& \varepsilon_{1}=k_{1} a_{1}, \varepsilon_{2}=k_{2} a_{2} \\
& X_{j}=\varepsilon_{j} x, T_{j}=\varepsilon_{j} t(j=1,2)
\end{aligned}
$$

where $k_{j}$ is the component (in $X_{j}$ direction) of the wave number vector of the carrier wave. $T_{j}$ and $X_{j}(j=1,2)$ are slow time and space scale, the group velocity $\vec{V}_{j}$ is

$$
\vec{V}_{j}=\partial \omega_{j} / \partial k_{j}(j=1,2)
$$

where $\omega_{j}$ is the frequency of the carrier wave of the wave group (see Figure 1)

The coefficients

$\alpha_{j}, \xi_{j}:$

$$
\alpha_{j}=\frac{1}{2} \frac{\partial^{2} \omega_{j}}{\partial k_{j}^{2}}, \xi_{j}=-\frac{2 k_{j}^{4}}{\omega_{j}}(j=1,2)
$$

$\zeta_{j}:$

$$
\begin{aligned}
& \zeta_{1}=-\frac{1}{2 g^{2}}\left[2 g \omega_{2} k_{1} k_{2}\left(k_{1}+2 k_{2}\right)+2 \omega_{1} k_{2}^{2} \omega_{2}^{2}\right] \\
& +\frac{G-J}{2 k_{1}}\left(\omega_{1} k_{1} k_{2}-\omega_{2} k_{1} k_{2}\right) \\
& +\frac{i F}{2}\left[k_{2}\left(k_{1}+k_{2}\right)+\frac{\omega_{2}}{\omega_{1}} k_{1}\left(k_{1}+k_{2}\right)-\kappa_{+} \frac{\omega_{1} \omega_{2}}{g}\right] \\
& -\frac{i H}{2}\left[k_{2}\left(k_{1}-k_{2}\right)+\frac{\omega_{2}}{\omega_{1}} k_{1}\left(k_{1}-k_{2}\right)-\kappa_{-} \frac{\omega_{1} \omega_{2}}{g}\right] \\
& \zeta_{2}=-\frac{1}{2 g^{2}}\left[2 g \omega_{1} k_{1} k_{2}\left(k_{2}+2 k_{1}\right)+2 \omega_{2} k_{1}^{2} \omega_{1}^{2}\right] \\
& +\frac{G-J}{2 K_{2}}\left(\omega_{2} k_{1} k_{2}-\omega_{1} k_{1} k_{2}\right) \\
& +\frac{i F}{2}\left[k_{1}\left(k_{1}+k_{2}\right)+\frac{\omega_{1}}{\omega_{2}} k_{2}\left(k_{1}+k_{2}\right)-\kappa_{+} \frac{\omega_{1} \omega_{2}}{g}\right] \\
& -\frac{i H}{2}\left[k_{1}\left(k_{1}-k_{2}\right)+\frac{\omega_{1}}{\omega_{2}} k_{2}\left(k_{1}-k_{2}\right)+\kappa_{-} \frac{\omega_{1} \omega_{2}}{g}\right]
\end{aligned}
$$

where

$$
\begin{aligned}
& F=-i\left\{k_{1} k_{2}\left(\omega_{1}+\omega_{2}\right)-\frac{\omega_{1} \omega_{2}}{g^{2}}\left(\omega_{1}+\omega_{2}\right)^{3}\right. \\
&\left.+\left[\omega_{1}\left(\kappa_{+}^{2}-k_{1}^{2}\right)+\omega_{2}\left(\kappa_{+}^{2}-k_{2}^{2}\right)\right]\right\} /\left[\omega_{+}^{2}-\left(\omega_{1}+\omega_{2}\right)^{2}\right] \\
& H=-i\left\{k_{1} k_{2}\left(\omega_{1}-\omega_{2}\right)+\frac{\omega_{1} \omega_{2}}{g^{2}}\left(\omega_{1}-\omega_{2}\right)^{3}\right. \\
&\left.+\left[\omega_{1}\left(\kappa_{-}^{2}-k_{1}^{2}\right)-\omega_{2}\left(\kappa_{-}^{2}-k_{2}^{2}\right)\right]\right\} /\left[\omega_{-}^{2}-\left(\omega_{1}-\omega_{2}\right)^{2}\right] \\
& G=\frac{1}{\omega_{+}^{2}-\left(\omega_{1}+\omega_{2}\right)^{2}}\left\{\left(\omega_{1}+\omega_{2}\right)\left[\frac{\omega_{1}}{g} k_{2}\left(k_{1}+k_{2}\right)+\frac{\omega_{2}}{g} k_{2}\left(k_{1}+k_{2}\right)\right]\right. \\
&\left.+\kappa_{+}\left[2 k_{1} k_{2}-\frac{\omega_{1} \omega_{2}}{g^{2}}\left(\omega_{1}+\omega_{2}\right)^{2}\right]\right\} \\
& J=\frac{-1}{\omega_{-}^{2}-\left(\omega_{1}-\omega_{2}\right)^{2}}\left\{\left(\omega_{1}-\omega_{2}\right)\left[\frac{\omega_{1}}{g} k_{2}\left(k_{1}-k_{2}\right)+\frac{\omega_{2}}{g} k_{2}\left(k_{1}-k_{2}\right)\right]\right. \\
&\left.+\kappa_{-}\left[2 k_{1} k_{2}-\frac{\omega_{1} \omega_{2}}{g^{2}}\left(\omega_{1}+\omega_{2}\right)^{2}\right]\right\} \\
& \omega_{+}=g \kappa_{+}, \omega_{-}=g \kappa_{-} \\
& \kappa_{+}=\left|k_{1}+k_{2}\right|, \kappa_{-}=\left|k_{1}-k_{2}\right|
\end{aligned}
$$

In deep water we have relation

$$
k_{1}=\omega_{1}^{2} / g, k_{2}=\omega_{2}^{2} / g
$$

Equation (1) is solved numerically. To compare the results with the results of linearity, we consider the linear superposition of these two wave groups. In the case of linear superposition (linear superposition of the four waves in Figure $1)$, the envelopes of the two wave groups are

$$
\begin{aligned}
& A_{1}^{\prime}(x, t)=a_{1} \cos \left(K_{1}\left(x-V_{1} t\right)\right) \\
& A_{2}^{\prime}(x, t)=a_{2} \cos \left(K_{2}\left(x-V_{2} t\right)\right)
\end{aligned}
$$

where

$$
\begin{gathered}
V_{1}=2 \omega_{1} / k_{1}, V_{2}=2 \omega_{2} / k_{2} \\
K_{1}=\left(k_{b}-k_{a}\right) / 2, K_{2}=\left(k_{d}-k_{c}\right) / 2
\end{gathered}
$$

The initial values of $\mathrm{A}_{1}, \mathrm{~A}_{2}, \mathrm{~A}_{1}^{\prime}, \mathrm{A}_{2}^{\prime}$ in equation (1) and (10) are

$$
\begin{aligned}
& A_{1}^{\prime}(x, 0)=A_{1}(x, 0)=a_{1} \cos \left(K_{1} x\right) \\
& A_{2}^{\prime}(x, 0)=A_{2}(x, 0)=a_{2} \cos \left(K_{2} x\right)
\end{aligned}
$$

where $a_{1}$ and $a_{2}$ are the amplitudes of the two wave groups.

In studying the interaction between the two wave groups we introduce a parameter:

$$
s=\frac{\Delta \omega_{2} \cdot s\left(\omega_{2}\right)}{\Delta \omega_{1} \cdot s\left(\omega_{1}\right)}
$$

where $\Delta \omega_{1}=\omega_{\mathrm{b}}-\omega_{\mathrm{a}}, \Delta \omega_{2}=\omega_{\mathrm{d}}-\omega_{\mathrm{c}}($ See Figure 1).

This parameter is found to be important in influencing the 
evolution of the envelope of the wave group.

\section{B. Results of Numerical Solution}

Now we consider the result of the evolution of the wave group envelope. The difference between the result of nonlinearity (the superposition of $\mathrm{A}_{1}$ and $\mathrm{A}_{2}$ ) and linearity (the superposition of $\mathrm{A}_{1}^{\prime}$ and $\mathrm{A}_{2}^{\prime}$ ) reveals the influence of nonlinearity on the height of the envelope. Suppose the height and average height of the envelope to be $h$ and $h_{0}$. We consider the probability of $h$ exceeding $2 h_{0}$. Let $F_{1}$ and $F_{n}$ represent the probability in the case of linearity and nonlinearity. The difference between $F_{1}$ and $F_{n}$ reflects the effect of nonlinearity on the height of the envelope.

We obtain $\left(F_{1}-F_{n}\right)$ for different values of $\Delta \omega_{1}$ and $\Delta \omega_{2}$. Figure 2 shows the relationship between $\left(F_{1}-F_{n}\right)$ and $s$ (The values of $\Delta \omega_{1}$ and $\Delta \omega_{2}$ are shown in Table 1$)$. The result shows that $\left(\mathrm{F}_{1}-\mathrm{F}_{\mathrm{n}}\right)$ increases with the increasing value of $\mathrm{s}$.
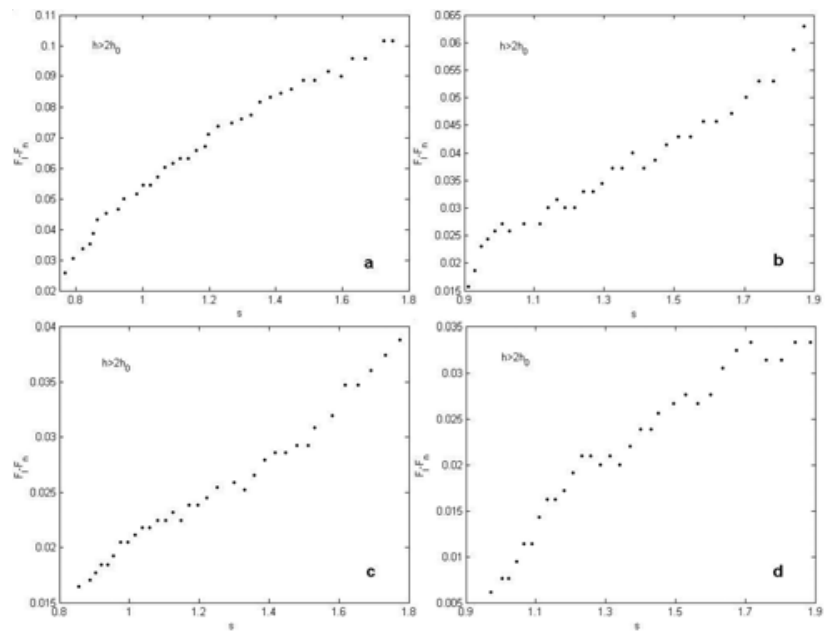

FIGURE II. THE RELATIONSHIP BETWEEN (Fl- Fn) AND s. WAVE STEEPNESS ak $0=0.1$

We now consider the probability of $h$ not exceeding $h_{0}$. Let $G_{1}$ and $G_{n}$ represent the probability in the case of linearity and nonlinearity, respectively. Figure 3 shows the relationship between $\left(\mathrm{G}_{\mathrm{n}}-\mathrm{G}_{1}\right)$ and $\mathrm{s}$ (The values of $\Delta \omega_{1}$ and $\Delta \omega_{2}$ are shown in Table 1). The result shows that $\left(\mathrm{G}_{\mathrm{n}}-\mathrm{G}_{\mathrm{l}}\right)$ increases with the increasing value of $\mathrm{s}$.
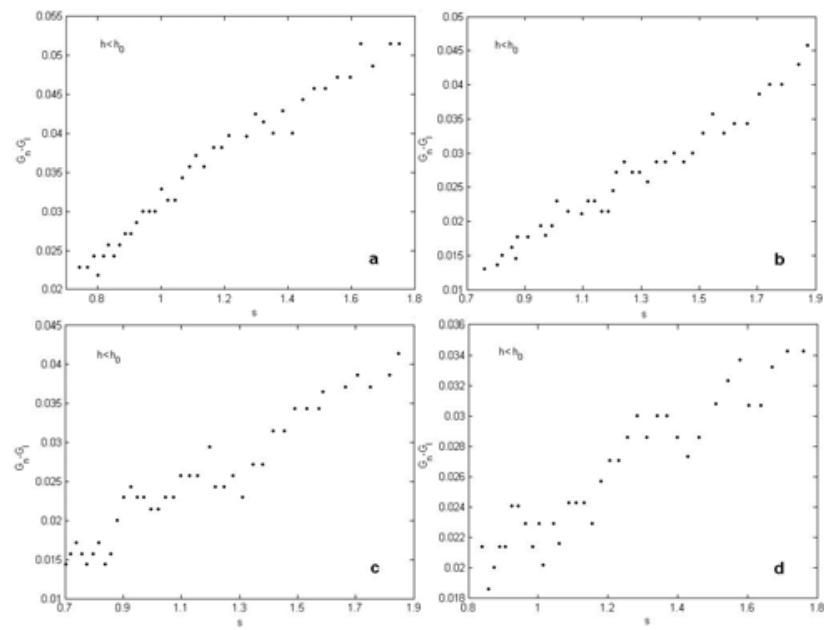

FIGURE III. THE RELATIONSHIP BETWEEN (Gn-Gl) AND s. WAVE STEEPNESS ak $0=0.1$

TABLE I. THE VALUES OF $\Delta \Omega_{1}$ AND $\Delta \Omega_{2}$ (IN FIGURES 2 AND 3)

\begin{tabular}{ccccc}
\hline & $\mathbf{a}$ & $\mathbf{b}$ & $\mathbf{c}$ & $\mathbf{d}$ \\
\hline$\Delta \omega_{1}$ & $0.13 \omega_{0}$ & $0.11 \omega_{0}$ & $0.078 \omega_{0}$ & $0.05 \omega_{0}$ \\
$\Delta \omega_{2}$ & $0.14 \omega_{0}$ & $0.12 \omega_{0}$ & $0.095 \omega_{0}$ & $0.06 \omega_{0}$ \\
\hline
\end{tabular}

The results in Figures 2 and 3 indicate that the nonlinear interaction between wave groups can influence the height of the envelope and the difference between linearity and nonlinearity increases with the values of $\mathrm{s}$. A consequence of the influence on the envelope is that in the case of nonlinearity the distribution of wave height is different from that in the case of linearity. This can be illustrated in Figure 4. For the superposition of the four wave components in Figure 1, the wave surface is the solid line in Figure 4. The dashed line is the envelope. $\mathrm{H}$ is wave height. Since in the case of nonlinearity the envelope is different from that in the case of linearity, the distributions of wave height in these two cases are obviously different.

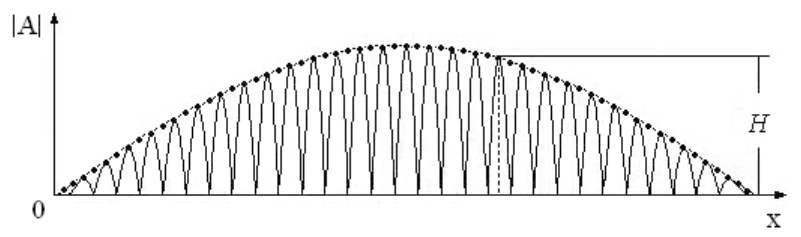

FIGURE IV. WAVE SURFACE AND ENVELOPE.

We can study the difference of wave height distribution in linearity and nonlinearity by studying the difference of the envelope in these two cases. We select $\mathrm{N}$ points (the dots) on the envelope and obtain the distribution of the height of the envelope. The distribution of the height of the envelope (distribution of the dots) can be considered as an approximation to the distribution of wave height (see Figure 4).

Figure 5 is the distribution of wave height obtained by this approximation, and Table 2 shows the values of $\mathrm{ak}_{0}$ and $\mathrm{s}$ used in Figure 5. The dots are the result of linearity. The small circles are the result of nonlinearity. Results in Figure 5 show 
that for waves with small and medium height $(H / \bar{H}<1.0)$, in the case of nonlinearity the curve of the distribution is higher than in the case of linearity. For waves with large height $(H / \bar{H}>1.5)$, in the case of nonlinearity the curve of the distribution is lower than in the case of linearity. $1.0<H / \bar{H}<1.5$ is the transition region.

A second result in Figure 5 is that the parameter $\mathrm{s}$ is an important factor in influencing wave height distribution. In Figures 5(a) and (b), wave steepness is the same. The value of $\mathrm{s}$ in Figure 5(b) is larger than that of $\mathrm{s}$ in Figure 5(a). The difference between linearity and nonlinearity in Figure 5(b) is larger than the difference in Figure 5(a). The result is the same in Figures 5(c) and (d) $\left(\mathrm{ak}_{0}=0.10\right)$, Figures 5(e) and (f) $\left(\mathrm{ak}_{0}=0.12\right)$ and other values of wave steepness. This result indicates that $\mathrm{s}$ is a measure of the effect of nonlinearity on wave height distribution. For large values of $s$, the deviation of the distribution from the result of linearity is larger (in fact this can be inferred from the results in Figures 2 and 3).

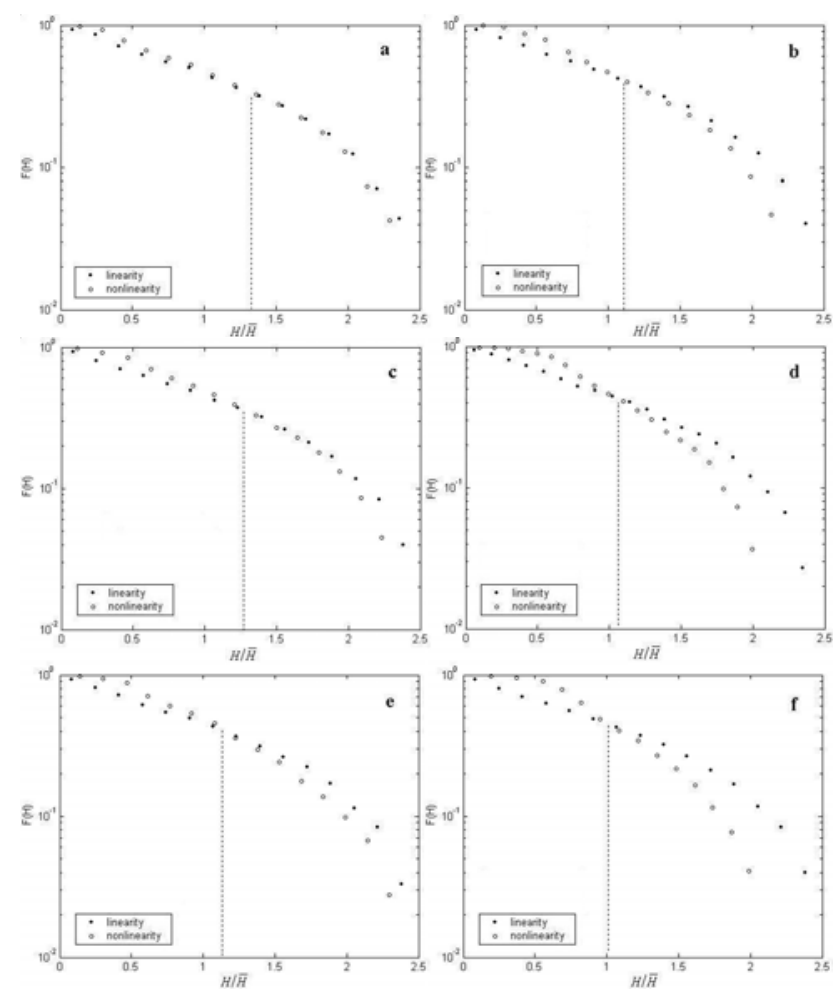

FIGURE V. DISTRIBUTION OF WAVE HEIGHT OBTAINED FROM THE ENVELOPE.

TABLE II. THE VALUES OF AK0 AND S (IN FIGURE 5)

\begin{tabular}{ccccccc}
\hline & $\mathbf{a}$ & $\mathbf{b}$ & $\mathbf{c}$ & $\mathbf{d}$ & $\mathbf{e}$ & $\mathbf{f}$ \\
\hline $\mathbf{a k} \mathbf{k}_{\mathbf{0}}$ & 0.08 & 0.08 & 0.10 & 0.10 & 0.12 & 0.12 \\
$\mathbf{s}$ & 1.26 & 1.78 & 1.13 & 1.67 & 1.05 & 1.43 \\
\hline
\end{tabular}

III. WIND WAVE EXPERIMENT RESULTS

In this section we study the distribution of wave height by wind wave experiment and compare the experimental results with the results in section II.

The experiment was made in the wind wave channel in the Laboratory of Physical Oceanography in Ocean University of China. The channel is 65 meters long with a rectangular cross-section of 1.2 meter in width and 1.4 meter in height. The test section is 50 meters long with a beach reducing wave reflection. The surface displacements of the waves were measured using surface-penetrating, capacitance-type gauges at ten fetches extending $7 \mathrm{~m}, 10.7 \mathrm{~m}, 14.35 \mathrm{~m}, 18 \mathrm{~m}, 21.7 \mathrm{~m}$, $25.4 \mathrm{~m}, 29.05 \mathrm{~m}, 32.75 \mathrm{~m}, 36.4 \mathrm{~m}$ and $41.1 \mathrm{~m}$ from the wind inlet section of the channel. Four mean wind speeds $U=4,6,8$, and $10 \mathrm{~m} / \mathrm{s}$ were chosen in the experiment.

In section II we studied the interaction of two wave groups. The superposition of two wave groups can be considered as a simple model for a wave field. This can be illustrated in Figure 6 . Figure 6 is a wind wave spectrum with peak frequency $\omega_{0}$.

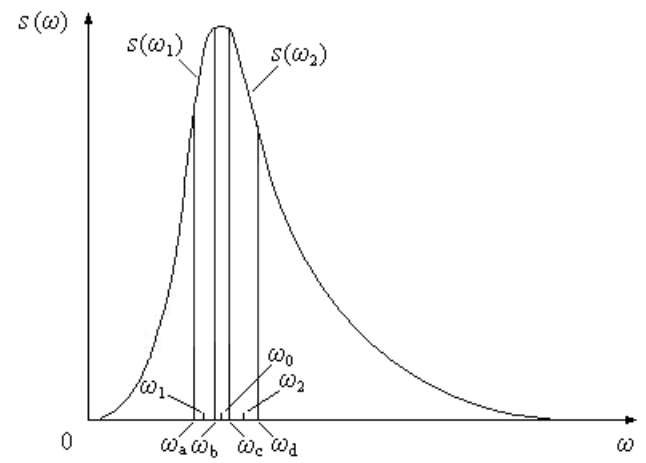

FIGURE VI. TWO WAVE GROUPS AS A SIMPLE MODEL FOR A SPECTRUM.

We consider two wave components with frequencies $\omega_{\mathrm{a}}=\omega_{1}-\mathrm{d} \omega_{1}$ and $\omega_{\mathrm{b}}=\omega_{1}+\mathrm{d} \omega_{1}$. The value of $\mathrm{d} \omega_{1}$ is very small. Since $\mathrm{d} \omega_{1}$ is very small, these two waves compose a wave group. Consider another two wave components with frequencies $\omega_{\mathrm{c}}=\omega_{2}-\mathrm{d} \omega_{2}$ and $\omega_{\mathrm{d}}=\omega_{2}+\mathrm{d} \omega_{2}$. The value of $\mathrm{d} \omega_{2}$ is very small. These two waves compose another wave group. In the same way we can find a third wave group in the wave spectrum. From this analysis we can see that wind waves can be considered as the superposition of many wave groups. The case of Figure 1 (two wave groups) in the theoretical study in this paper can be considered as a simple model for a spectrum. In this section we will see that the wave height distribution results in two wave groups in section II are consistent with the experimental results of wind waves.

\section{A. Distribution of Wave Height}

Figure 7 is the distribution of wave height for wind waves (the values of Fetch and wind speed are shown in Table 3). The dots are the experimental result. The solid line is the Rayleigh distribution.

From Figure 7 we can see that for waves with small and medium height $(H / \bar{H}<1.0)$, the curve of the distribution in experimental result is higher than the curve of Rayleigh distribution. For waves with large height $(H / \bar{H}>1.5)$, the curve of the distribution in experimental result is lower than the curve of Rayleigh distribution. The transition region is 
$1.0<H / \bar{H}<1.5$. These results are consistent with the theoretical results for two wave groups in Figure 5. In Figure 7 the Rayleigh distribution (derived on the basis of linearity) corresponds to dots (cases of linear superposition of the wave components) in Figure 5. The dots (experimental results) in Figure 7 corresponds to small circles (cases of nonlinearity) in Figure 5. In Figure 5 for waves with small and medium height $(H / \bar{H}<1.0)$, in the case of nonlinearity the curve of the distribution is higher than in the case of linearity. For waves with large height $(H / \bar{H}>1.5)$, in the case of nonlinearity the curve of the distribution is lower than in the case of linearity. The transition region is $1.0<H / \bar{H}<1.5$. All these results are in consistency with the results in Figure 7.
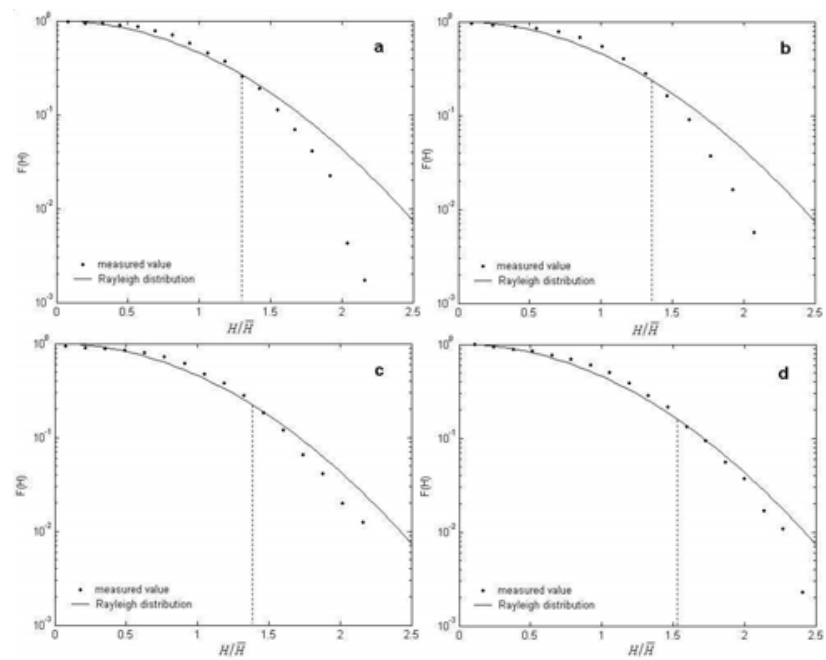

FIGURE VII. EXPERIMENTAL RESULT OF THE DISTRIBUTION OF WAVE HEIGHT.

TABLE III. THE VALUES OF FETCH AND WIND SPEED (IN FIGURE 7)

\begin{tabular}{ccccc}
\hline & a & b & c & d \\
\hline Fetch & $41.00 \mathrm{~m}$ & $36.40 \mathrm{~m}$ & $29.05 \mathrm{~m}$ & $14.35 \mathrm{~m}$ \\
$\begin{array}{l}\text { Wind } \\
\text { Speed }\end{array}$ & $10 \mathrm{~m} / \mathrm{s}$ & $8 \mathrm{~m} / \mathrm{s}$ & $10 \mathrm{~m} / \mathrm{s}$ & $6 \mathrm{~m} / \mathrm{s}$ \\
\hline
\end{tabular}

B. Influence of Spectral Shape on Wave Height Distribution

A parameter s is introduced in (14). From Figure 1 we can see that this parameter $\mathrm{s}$ is the ratio of the right rectangle area to the left rectangle area. When Figure 1 is considered as a simple model of a wave spectrum, the right rectangle is the region on the right side of the peak frequency (hereafter referred to as "right region"). The left rectangle is the region on the left side of the peak frequency (hereafter referred to as "left region"). $s$ is the ratio of right region area to left region area.

For a continuous wind wave spectrum, the right region area $s_{2}$ and left region area $s_{1}$ can be obtained according to the peak frequency of the spectrum. On analogy of (14), we can introduce a parameter $\mathrm{S}$ for a continuous spectrum

$$
S=S_{2} / s_{1}
$$

$\mathrm{S}$ corresponds to $\mathrm{s}$ in section II. From the wind wave spectra measured in the experiment we obtain the values of $S$ in these spectra and study the influence of $S$ on the distribution of wave height.

Figure 8 is the distribution of wave height for waves with different values of $\mathrm{S}$ (the values of the parameters are shown in Table 4). The dots are cases of small values of S. The small circles are cases of large values of $\mathrm{S}$. The solid line is Rayleigh distribution.
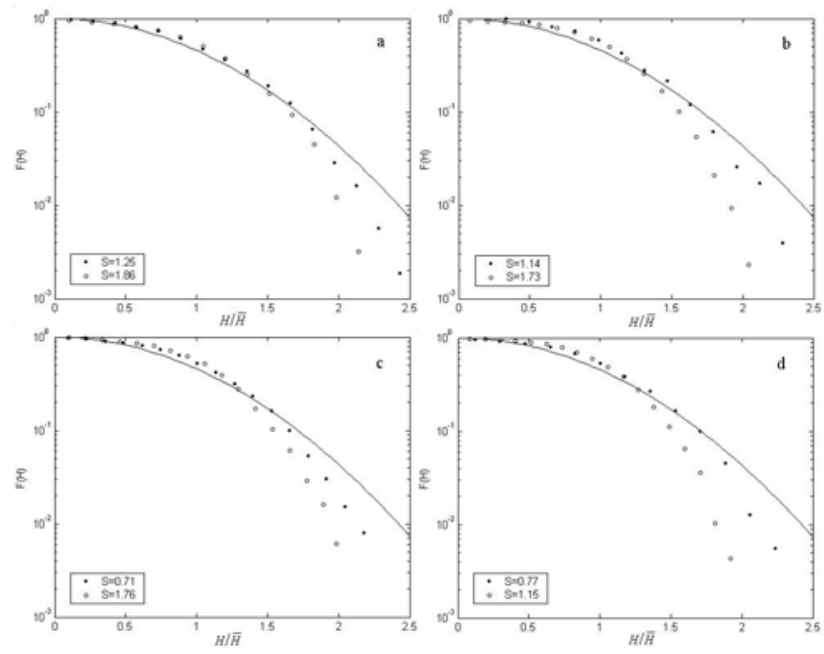

FIGURE VIII. DISTRIBUTION OF WAVE HEIGHT FOR DIFFERENT VALUES OF $\mathrm{S}$.

From Figure 8 we can see that when wave steepness is the same, for large values of $\mathrm{S}$ the deviation of experimental result from Rayleigh distribution is larger. This is consistent with the results in Figure 5. The parameter S corresponds to parameter $\mathrm{s}$ in Figure 5. Results in Figure 5 show that when wave steepness is the same, for large values of $s$ the deviation of the distribution from the result of linearity (corresponding to Rayleigh distribution in Figure 8) is larger.

TABLE IV. THE VALUES OF AK0, FETCH, WIND SPEED AND S (IN FIGURE 8)

\begin{tabular}{|c|c|c|c|c|c|c|c|}
\hline & \multirow[b]{2}{*}{$\mathbf{a k}_{0}$} & \multicolumn{3}{|c|}{ dots } & \multicolumn{3}{|c|}{ circles } \\
\hline & & Fetch & $\begin{array}{l}\text { wind } \\
\text { speed }\end{array}$ & S & Fetch & $\begin{array}{l}\text { wind } \\
\text { speed }\end{array}$ & $\mathbf{S}$ \\
\hline $\mathbf{a}$ & 0.08 & $41.00 \mathrm{~m}$ & $6 \mathrm{~m} / \mathrm{s}$ & 1.25 & $21.70 \mathrm{~m}$ & $10 \mathrm{~m} / \mathrm{s}$ & 1.86 \\
\hline b & 0.10 & $14.35 \mathrm{~m}$ & $4 \mathrm{~m} / \mathrm{s}$ & 1.14 & $32.75 \mathrm{~m}$ & $10 \mathrm{~m} / \mathrm{s}$ & 1.73 \\
\hline c & 0.11 & $25.40 \mathrm{~m}$ & $8 \mathrm{~m} / \mathrm{s}$ & 0.71 & $29.05 \mathrm{~m}$ & $10 \mathrm{~m} / \mathrm{s}$ & 1.76 \\
\hline d & 0.12 & $21.70 \mathrm{~m}$ & $8 \mathrm{~m} / \mathrm{s}$ & 0.77 & $41.00 \mathrm{~m}$ & $10 \mathrm{~m} / \mathrm{s}$ & 1.15 \\
\hline
\end{tabular}

The result in Figure 8 indicates that the ratio of right region area to left region area in a spectrum is an important factor in influencing the distribution of wave height. This implies that the shape of the spectrum is important in influencing the distribution of wave height. The theoretical result in Figure 5 gives an explanation to this. The non-linear interactions between wave groups can cause the deviation of wave height distribution from Rayleigh distribution. The parameter $s$ in (14) is a measure of the effect of nonlinear interaction between two wave groups. This parameter 
corresponds to the ratio of right region area to left region area in a continuous spectrum. The ratio $\mathrm{S}$ in (15) is a measure of nonlinearity in wave group interactions in a continuous spectrum. For two continuous spectra with different spectral shape, the values of $\mathrm{S}$ are different. The effects of nonlinearity in these two continuous spectra are different. This gives rise to the difference in wave height distribution.

\section{Wave Height Distribution for Simulated Waves}

We now use the measured spectra to simulate wind waves and study the distribution of wave height. The wave surface displacement can be expressed a

$$
\zeta(t)=2 \int_{0}^{\infty} \sqrt{S(f) d f} \cos (2 \pi f t+\theta)
$$

where $\zeta$ is wave surface displacement, $\mathrm{S}(\mathrm{f})$ is spectrum, $\mathrm{f}$ is frequency and $\theta$ is the phase which is distributed randomly from 0 to $2 \pi$.

We use the measured wind wave spectra in the experiment to simulate $\zeta$ according to (16). The simulated wave surface displacement is linear superposition of different frequency waves. From the simulated wave surface displacement we can obtain wave height distribution.

The results in Figure 9 show clearly the effect of nonlinearity on the distribution of wave height (the values of Fetch and wind speed are shown in Table 5). In Figure 9 the dots are wave height distribution obtained from the measured wave surface displacement. The small circles are wave height distribution obtained from the simulated wave surface displacement. In each figure the spectrum of the simulated waves is the same as the spectrum of the measured waves. The dashed line is Rayleigh distribution. Since the simulated wave surface displacement is the result of linear superposition, the deviations of the dots from small circles are obviously caused by nonlinearity.
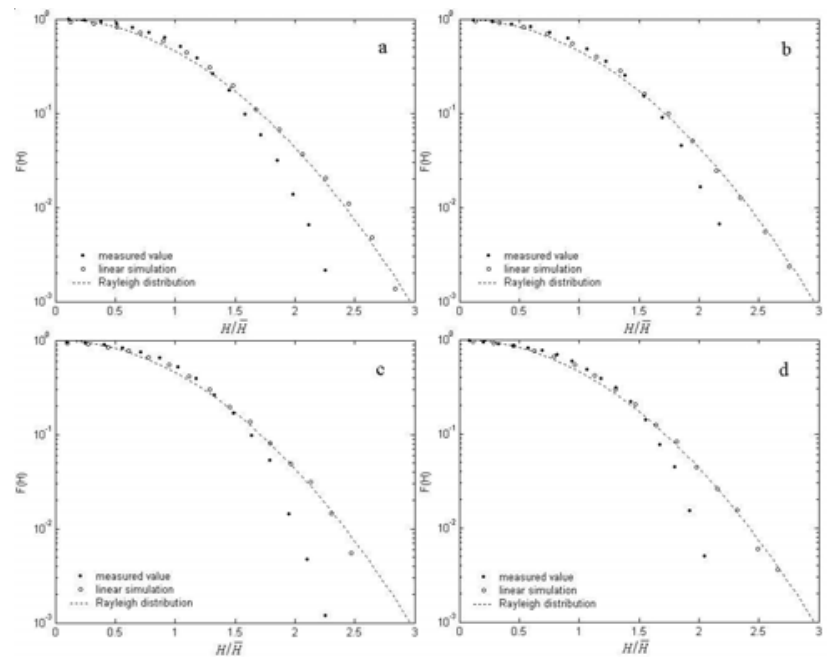

FIGURE IX. DISTRIBUTION OF WAVE HEIGHT FOR SIMULATED WAVES AND MEASURED WAVES.
TABLE V. THE VALUES OF FETCH AND WIND SPEED (IN FIGURE 9)

\begin{tabular}{ccccc}
\hline & a & b & c & d \\
\hline Fetch & $7 \mathrm{~m}$ & $14.35 \mathrm{~m}$ & $21.7 \mathrm{~m}$ & $25.4 \mathrm{~m}$ \\
$\begin{array}{r}\text { Wind } \\
\text { Speed }\end{array}$ & $10 \mathrm{~m} / \mathrm{s}$ & $8 \mathrm{~m} / \mathrm{s}$ & $10 \mathrm{~m} / \mathrm{s}$ & $10 \mathrm{~m} / \mathrm{s}$ \\
\hline
\end{tabular}

\section{CONCLUSIONS}

In this paper we studied the effect of wave group interaction on the distribution of wave height. We found that the interaction of two wave groups may cause the deviation of wave height distribution from the result of linear theory. For low and medium wave heights, in the case of nonlinearity the distribution curve is higher than in the case of linearity. For waves with large height, in the case of nonlinearity the distribution curve is lower than in the case of linearity. The theoretical result is in consistency with the result of wind wave experiment. A parameter reflecting the spectral shape was introduced in studying the influence of wave group interaction on the distribution of wave height. It is a measure of the effect of nonlinearity and is found to be important in influencing wave height distribution.

\section{ACKNOWLEDGMENTS}

Partial support for this research was provided by the National Key Research and Development Plan through grant 2016YFC1402304, the Key Research and Development Plan of Shandong Province through grant 2016ZDJS09A02, the National Natural Science Foundation of China through grant 41606006.

\section{REFERENCES}

[1] M. S. Longuet-Higgins, "The statistical analysis of a random moving surface,” Philos. Trans. Roy. Soc., vol. 249, pp. 321-387, 1957.

[2] G. Z. Forristall, "On the statistical distribution of wave heights in a storm,” J. Geophys. Res. , vol. 83, pp. 2353-2358, 1978.

[3] G. Z. Forristall, "Wave crest distributions: observations and second-order theory," J. Phys. Oceanogr. , vol. 30, pp. 1931-1943, 2000.

[4] M. A. Tayfun, "Distribution of crest-to-trough wave heights," J. Waterway, Port, Coastal, Ocean Eng. , vol. 107, pp. 149-158, 1981.

[5] Y. J. Hou, Y. L. Duan, G. X. Chen, P. Qi, and G. C. Si, "Statistical distribution of nonlinear random wave height in shallow water," Sci. China Ser. D-Earth Sci., vol. 39, pp. 1795-1800, 2009. (in Chinese)

[6] P. G. Petrova, C. G. Soares, "Wave height distributions in bimodal sea states from offshore basins," Ocean Eng., vol. 38, pp. 658-672, 2011.

[7] H. D. Zhang, C. G. Soares, M. Onorato, "Modelling of the spatial evolution of extreme laboratory wave Heights with the nonlinear Schrödinger and Dysthe equations," Ocean Eng., vol. 89, pp. 1-9, 2014.

[8] V. E. Zakhraov, "Stability of periodic waves of finite amplitude on the surface of a deep fluid,” J. Appl. Mech. Tech. Phys., vol. 4, , pp. 86-90, 1968.

[9] J. L. Hammack, D. M. Henderson, and H. Segur, "Progressive waves with persistent two-dimensional surface patterns in deep water," J. Fluid Mech., vol. 532, pp. 1-52, 2005.

[10] P. K. Shukla, I. Kourakis, B. Eliasson, M. Marklund, and L. Stenflo, "Instability and Evolution of Nonlinearly Interacting Water Waves," Phys. Rev. Lett., vol. 97, 094501, 2006.

[11] M. Onorato, A. R. Osborne, and M. Serio, "Modulational Instability in Crossing Sea States: A Possible Mechanism for the Formation of Freak Waves,” Phys. Rev. Lett., vol. 96, 014503, 2006.

[12] T. Waseda, T. Kinoshita, and H. Tamura, "Evolution of a Random Directional Wave and Freak Wave Occurrence," J. Phys. Oceanogr., vol. 
39, pp. 621-639, 2009.

[13] T. Waseda, T. Kinoshita, L. Cavaleri, and A. Toffoli, "Third-order resonant wave interactions under the influence of background current fields,” J. Fluid Mech., vol. 784, pp. 51-73, 2015. 